\title{
Gravitational lensing statistics with extragalactic surveys
}

\author{
II. Analysis of the Jodrell Bank-VLA Astrometric Survey \\ P. Helbig ${ }^{1}$, D. Marlow ${ }^{1, \star}$, R. Quast ${ }^{2}$, P.N. Wilkinson ${ }^{1}$, I.W.A. Browne ${ }^{1}$, and L.V.E. Koopmans ${ }^{3}$ \\ 1 University of Manchester, Nuffield Radio Astronomy Laboratories, Jodrell Bank, Macclesfield, Cheshire SK11 9DL, UK \\ ${ }^{2}$ Universität Hamburg, Hamburger Sternwarte, Gojenbergsweg 112, D-21029 Hamburg, Germany \\ 3 University of Groningen, Kapteyn Astronomical Institute, Postbus 800, NL-9700 AV Groningen, The Netherlands
}

Received December 2, 1998; accepted January 12, 1999

\begin{abstract}
We present constraints on the cosmological constant $\lambda_{0}$ from gravitational lensing statistics of the Jodrell Bank-VLA Astrometric Survey (JVAS). Although this is the largest gravitational lens survey which has been analysed, cosmological constraints are only comparable to those from optical surveys. This is due to the fact that the median source redshifts of JVAS are lower, which leads to both relatively fewer lenses in the survey and a weaker dependence on the cosmological parameters. Although more approximations have to be made than is the case for optical surveys, the consistency of the results with those from optical gravitational lens surveys and other cosmological tests indicate that this is not a major source of uncertainty in the results. However, joint constraints from a combination of radio and optical data are much tighter. Thus, a similar analysis of the much larger Cosmic Lens All-Sky Survey should provide even tighter constraints on the cosmological constant, especially when combined with data from optical lens surveys.
\end{abstract}

At $95 \%$ confidence, our lower and upper limits on $\lambda_{0}-\Omega_{0}$, using the JVAS lensing statistics information alone, are respectively -2.69 and 0.68 . For a flat universe, these correspond to lower and upper limits on $\lambda_{0}$ of respectively -0.85 and 0.84 . Using the combination of JVAS lensing statistics and lensing statistics from the literature as discussed in Quast \& Helbig (1999) the corresponding $\lambda_{0}-\Omega_{0}$ values are -1.78 and 0.27 . For a flat universe, these correspond to lower and upper limits on $\lambda_{0}$ of respectively -0.39 and 0.64 .

Send offprint requests to: P. Helbig

* Present Address: University of Pennsylvania, Dept. of Physics and Astronomy, 209 S. $33^{\text {rd }}$ Street, Philadelphia, PA 19104-6396, U.S.A.

Correspondence to: p.helbig@jb.man.ac.uk
Key words: gravitational lensing - cosmology: theory cosmology: observations

\section{Introduction}

The use of gravitational lensing statistics as a cosmological tool was first considered in detail by Turner et al. (1984); the influence of the cosmological constant was investigated thoroughly by Fukugita et al. (1992), building on the work of Turner (1990) and Fukugita et al. (1990). Kochanek (1996a, and references therein) and, more recently, Falco et al. (1998, hereafter FKM) have laid the groundwork for using gravitational lensing statistics for the detailed analysis of extragalactic surveys. Quast \& Helbig (1999, hereafter Paper I) reanalysed optical surveys from the literature, for the first time exploring a range of the $\lambda_{0}-\Omega_{0}$ parameter space large enough to enable a comparison with other cosmological tests. Here, we use the formalism outlined in Paper I to analyse the Jodrell Bank-VLA Astrometric Survey (JVAS), the largest completed gravitational lens survey to date.

Radio surveys offer several advantages over optical surveys (see, e.g., FKM): one doesn't have to worry about systematic errors due to extinction or a lens galaxy of apparent brightness comparable to that of the lensed images of the source, the resolution (of followup observations if not of the survey proper) is much smaller than the typical image separation, parent catalogues in the form of large-area surveys exist from which unbiased samples can be selected and relatively easily observed. Disadvantages in the radio are due to our relatively poor knowledge of the flux density-dependent redshift distribution or equivalently the redshift-dependent number-magnitude relation.

For a description of our method see Paper I. The plan of this paper is as follows. Section 2 describes the JVAS 
gravitational lens survey. In Sect. 3 we describe the calculations we have done based on the JVAS data. Section 4 presents our results, using both the JVAS data alone and in combination with the results from the optical surveys analysed in Paper I. Finally in Sect. 5 we compare our results to those of Paper I and present our conclusions and our prognosis for the analysis of future large surveys such as CLASS.

\section{The JVAS gravitational lens survey}

\subsection{The sample}

The Jodrell Bank-VLA Astrometric Survey (JVAS) is a survey for flat-spectrum radio sources with a flux density greater than $200 \mathrm{mJy}$ at $5 \mathrm{GHz}$. Flat-spectrum radio sources are likely to be compact, thus making it easy to recognise the lensing morphology. In addition, they are likely to be variable, making it possible to determine $H_{0}$ by measuring the time delay between the lensed images. (See Biggs et al. 1998, for the description of a time delay measurement in a JVAS gravitational lens system.) JVAS is also a survey for MERLIN phase-reference sources and as such is described in Patnaik et al. (1992), Browne et al. (1998) and Wilkinson et al. (1998). JVAS as a gravitational lens survey, the lens candidate selection, followup process, confirmation criteria and a discussion of the JVAS gravitational lenses is described in detail in King et al. (1999) (see also King \& Browne 1996).

In order to have a parent sample which is as large as possible and as cleanly defined as practical, our "JVAS gravitational lens survey sample" is slightly different than the "JVAS phase-reference calibrator sample". For the former, the source must be a point source and must have a good starting position (so that the observation was correctly pointed) while its precise spectral index is not important. For the latter, only the spectral index is important, as the source can be slightly resolved or the observation can be less than perfectly pointed. Thus, the JVAS astrometric sample (Patnaik et al. 1992; Browne et al. 1998; Wilkinson et al. 1998) contains 2144 sources. To these must be added 103 sources which were too resolved to be used as phase calibrators and 61 sources which had bad starting positions (thus the observations were too badly pointed to be useful for the astrometric sample), bringing the total to 2308. This formed our gravitational lens sample, since these additional sources were also searched for gravitational lenses (King et al. 1999) (none were found meeting the JVAS selection criteria).

\subsection{The lenses}

We have used the gravitational lens systems in Table 1 in this analysis. The JVAS lens B1938+666 (King et al. 1998) was not included because it is not formally a part of the sample, having a too steep spectral index and having been recognised on the basis of a lensed extended source as opposed to lensed compact components. Also, the JVAS lens B2114+022 (Augusto et al. 1999) was not included because it is not a single-galaxy lens system.

\section{Calculations}

A major difference between the analysis of an optical survey (see Paper I and references therein) and a radio survey is that in the latter one does not know the redshifts of all the unlensed sources. One can still use the formalism of Paper I, however, substituting for the non-lensed objects in the sample a subsample with known redshifts, multiplying the logarithm of this contribution from the non-lenses to the likelihood by the ratio of the size of the parent sample to that of the subsample. Alternatively, one can take the redshifts from a sample selected according to the same criteria, assigning these randomly to objects in (a subsample of) the parent sample for a similar flux density range. Similarly, one does not know the number-magnitude relation for the sample and for its extension to fainter flux densities (needed to allow for the lens amplification). Again, this can be estimated from either a subsample (through extrapolation) or from another sample selected according to the same criteria (either through extrapolation or by having a fainter flux density limit in this other sample; in the latter case obviously the selection criteria should be identical to that of the original sample except for the lower flux density limit).

For this analysis, due to the paucity of the observational data, we have made rather stark assumptions: the redshift distribution of the sample is assumed to be identical to that of the CJF sample (Taylor et al. 1996), independent of flux density, and the number-magnitude relation is assumed to be identical to that of CLASS (Cosmic Lens All-Sky Survey, Myers et al. 1999), independent of redshift.

Otherwise, we have followed the procedure outlined in Paper I, calculating the a priori likelihood of obtaining the observational data as a function of $\lambda_{0}$ and $\Omega_{0}$ and the a posteriori likelihood for the three different choices of prior information used in Paper I. We present results both for the JVAS lens survey and for the combination of the JVAS results with those from the optical surveys analysed in Paper I.

\section{Results and discussion}

The left panel of Fig. 1 shows the constraints on the cosmological parameters $\lambda_{0}$ and $\Omega_{0}$ based only on the information obtained from the JVAS lens statistics, while the right panel shows the joint constraints from the JVAS lens sample and the optical samples from Paper I. Figure 2 
Table 1. JVAS lenses used in this analysis. Of the information in the table, for this analysis we use only the source redshift $z_{\mathrm{s}}$ and the image separation $\Delta \theta$

\begin{tabular}{lllll}
\hline \hline Name & \# images & $\Delta \theta\left[^{\prime \prime}\right]$ & $z_{1}$ & lens galaxy \\
\hline B0218+357 & $2+$ ring & 0.334 & 0.6847 & 0.96 \\
MG0414+054 & 4 & 2.09 & 0.9584 & 2.639 \\
B1030+074 & 2 & 1.56 & 0.599 & 1.535 \\
B1422+231 & 4 & 1.28 & 0.337 & elliptical \\
\hline
\end{tabular}
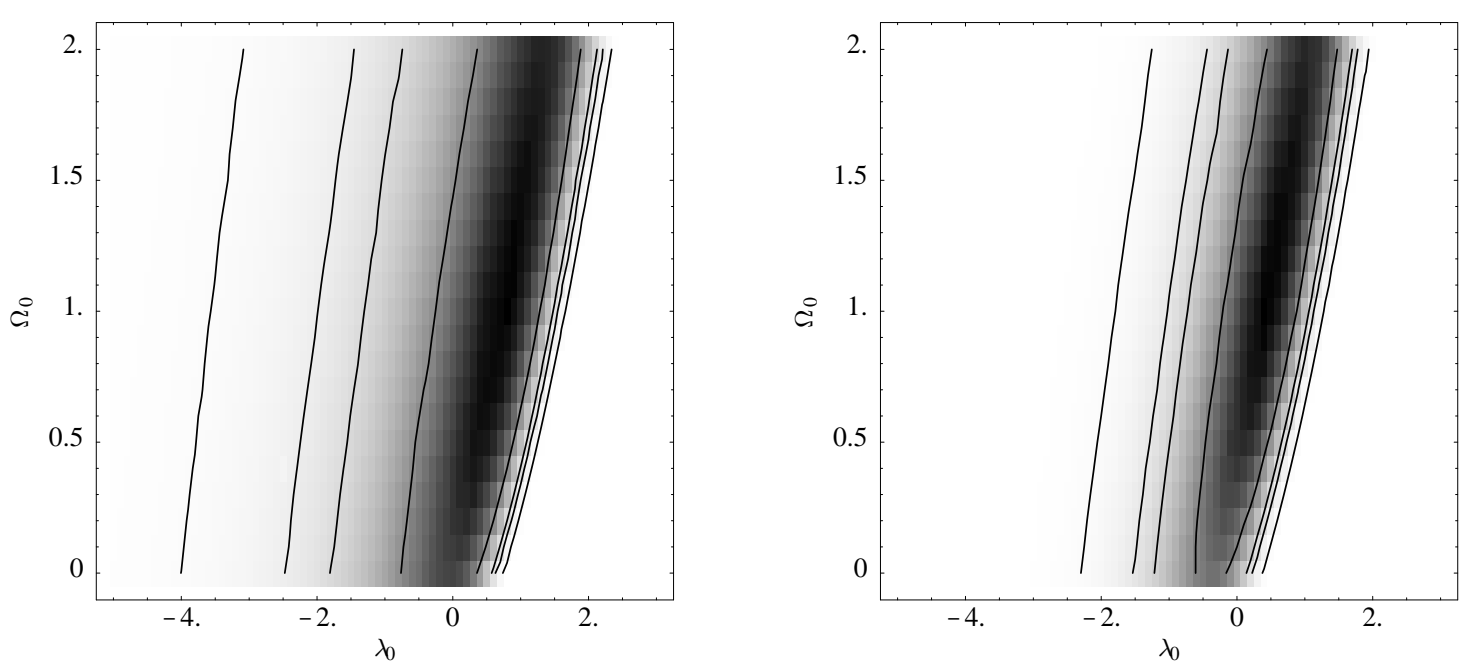

Fig. 1. Left panel: The likelihood function $p\left(D \mid \lambda_{0}, \Omega_{0}, \boldsymbol{\xi}_{0}\right)$ based on the JVAS lens sample. All nuisance parameters are assumed to take precisely their mean values. The pixel grey level is directly proportional to the likelihood ratio, darker pixels reflect higher ratios. The pixel size reflects the resolution of our numerical computations. The contours mark the boundaries of the minimum 0.68, 0.90, 0.95 and 0.99 confidence regions for the parameters $\lambda_{0}$ and $\Omega_{0}$. Right panel: Exactly the same as the left panel, but the joint likelihood from the JVAS lens sample and the optical samples from Quast \& Helbig (1999, Paper I)

is identical except that one of the input parameters, the normalisation of the galaxy luminosity function, was increased by two standard deviations. This gives an idea of the magnitude of systematic uncertainties. (See the discussion in Paper I.)

The left plot in the top row of Fig. 3 shows the joint likelihood of our lensing statistics analysis and that obtained by using conservative estimates for $H_{0}$ and the age of the universe (see Paper I). Although neither method alone sets useful constraints on $\Omega_{0}$, their combination does, since the constraint from $H_{0}$ and the age of the universe only allows large values of $\Omega_{0}$ for $\lambda_{0}$ values which are excluded by lens statistics. Even though the $68 \%$ confidence contour still allows almost the entire $\Omega_{0}$ range, it is obvious from the grey scale that much lower values of $\Omega_{0}$ are favoured by the joint constraints. The upper limit on $\lambda_{0}$ changes only slightly while, as is to be expected, the lower limit becomes tighter. Right plot: exactly the same, but including optical constraints from Paper I. The upper limits on $\lambda_{0}$ decrease slightly, while the lower limits improve considerably. The latter is probably due to the fact that, in addition to just using more data the JVAS sources are at significantly different redshifts than those from the optical surveys analysed in Paper I (the JVAS sources are generally at lower redshift). The former is consistent with the slightly higher optical depth for radio surveys found by FKM and will be discussed more below.

The middle row of Fig. 3 shows the effect of including our prior information on $\Omega_{0}$ (see Paper I). As is to be expected, (for both the JVAS and combination data sets) lower values of $\Omega_{0}$ are favoured. This has the side effect of weakening our lower limit on $\lambda_{0}$ (though only slightly affecting the upper limit). This should not be regarded as a weakness, however, since including prior information for $\lambda_{0}$ and $\Omega_{0}$ from the constraint from $H_{0}$ and the age of the universe as well as for $\Omega_{0}$ itself, as illustrated in the bottom row of Fig. 3, tightens the lower limit again (without appreciably affecting the upper limit).

We believe that the right plot of the bottom row of Fig. 3 represents very robust constraints in the $\lambda_{0}-\Omega_{0}$ plane. The upper limits on $\lambda_{0}$ come from gravitational lensing statistics, which, due to the extremely rapid increase in the optical depth for larger values of $\lambda_{0}$, are quite robust and relatively insensitive to uncertainties in the input data (cf. Fig. 2 and the discussion of the effect of changing the most uncertain input parameter by $2 \sigma$ in Paper I) as well as to the prior information used (compare the upper, lower and middle rows of Fig. 3). The combination of data from JVAS and optical surveys leads to much tighter lower limits on $\lambda_{0}$ than using either alone. 

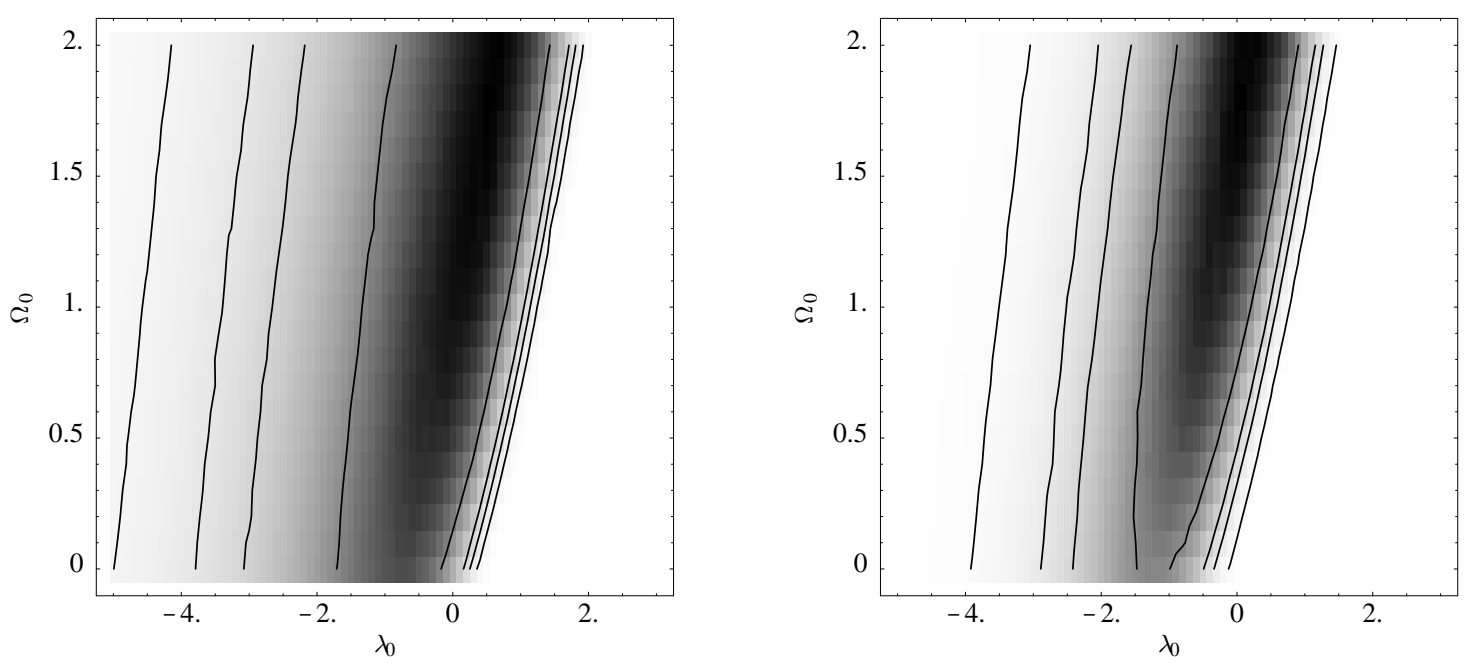

Fig. 2. Exactly the same as Fig. 1, but the parameter $n_{\mathrm{e}}$ is increased by two standard deviations. This parameter, the normalisation of the luminosity function of the lens galaxies, is one of the more uncertain input parameters, thus one can get a rough estimate of the overall uncertainty by comparing this figure and Fig. 1. See the discussion in Paper I

The upper and lower limits on $\Omega_{0}$ are based on a number of different methods and appear to be quite robust (see Paper I). The combination of the relatively secure knowledge of $H_{0}$ and the age of the universe combine with lens statistics to produce a good lower limit on $\lambda_{0}$, although this is to some extent still subject to the caveats mentioned above.

If one is interested in the allowed range of $\lambda_{0}$, one can marginalise over $\Omega_{0}$ to obtain a probability distribution for $\lambda_{0}$. This is illustrated in Fig. 4 and Table 2 .

The comparison values from this work corresponding to those in Tables 3 and 4 of Paper I are presented in Tables 3 and 4.

For a "likely" $\Omega_{0}$ value of 0.3 we have calculated the likelihood with the higher resolution $\Delta \lambda_{0}=0.01$. This is show in Fig. 5. From these calculations one can extract confidence limits which, due to the higher resolution in $\lambda_{0}$, are more accurate. These are presented in Table 5 and should be compared to those for $p\left(D \mid \lambda_{0}\right)$ from Table 3 .

As mentioned in Paper I, to aid comparisons with other cosmological tests, the data for the figures shown in this paper are available at

http://multivac.jb.man.ac.uk:8000/ceres

/data_from_papers/JVAS.html

and we urge our colleagues to follow our example.

\section{Conclusions and outlook}

We have used the method outlined in Quast \& Helbig (1999) to measure the cosmological constant $\lambda_{0}$ from the lensing statistics of the Jodrell Bank-VLA Astrometric Survey. At 95\% confidence, our lower and upper limits on $\lambda_{0}-\Omega_{0}$, using the JVAS lensing statistics information alone, are respectively -2.69 and 0.68 . For a flat universe, these correspond to lower and upper limits on $\lambda_{0}$ of respectively -0.85 and 0.84 . Using the combination of JVAS lensing statistics and lensing statistics from the literature as discussed in Quast \& Helbig (1999) the corresponding $\lambda_{0}-\Omega_{0}$ values are -1.78 and 0.27 . For a flat universe, these correspond to lower and upper limits on $\lambda_{0}$ of respectively -0.39 and 0.64 . Note that the lower limit is affected more than the upper limit with respect to the difference between the JVAS results and those in Paper I and with respect to combining the JVAS results with those from Paper I.

Our determination is consistent with other recent measurements of $\lambda_{0}$, both from lensing statistics and from other cosmological tests (see Quast \& Helbig 1999, Paper I, for a discussion). We confirm the result of Falco et al. (1998, FKM) that radio surveys give higher values of $\lambda_{0}$ than optical surveys. Cooray et al. (1999) and Cooray (1999) obtain a $95 \%$ confidence upper limit on $\lambda_{0}$ in a flat universe of 0.79 from analyses of the Hubble Deep Field and CLASS. However, these analyses suffer from systematic effects due to our ignorance of the underlying flux density-dependent redshift distribution (or, equivalently, the redshift-dependent luminosity function) of the unlensed parent population. As discussed in Cooray (1999), the value of $\lambda_{0}$ obtained from CLASS will decrease if the mean redshift of the sample is lower than presumed. Thus, although there is no real conflict at present as the lower limits on $\lambda_{0}$ are not as tight, it seems not unlikely that a more detailed analysis of CLASS, incorporating more information about the unlensed parent population, will result in a value more in line with our value obtained from the JVAS analysis. Of course, the JVAS analysis also suffers from systematic effects, but the general agreement between the results obtained from the analysis of optical surveys (cf. Paper I and references therein) and radio surveys as presented here and in FKM suggests that these are not overwhelming. Also, the difference, a higher 

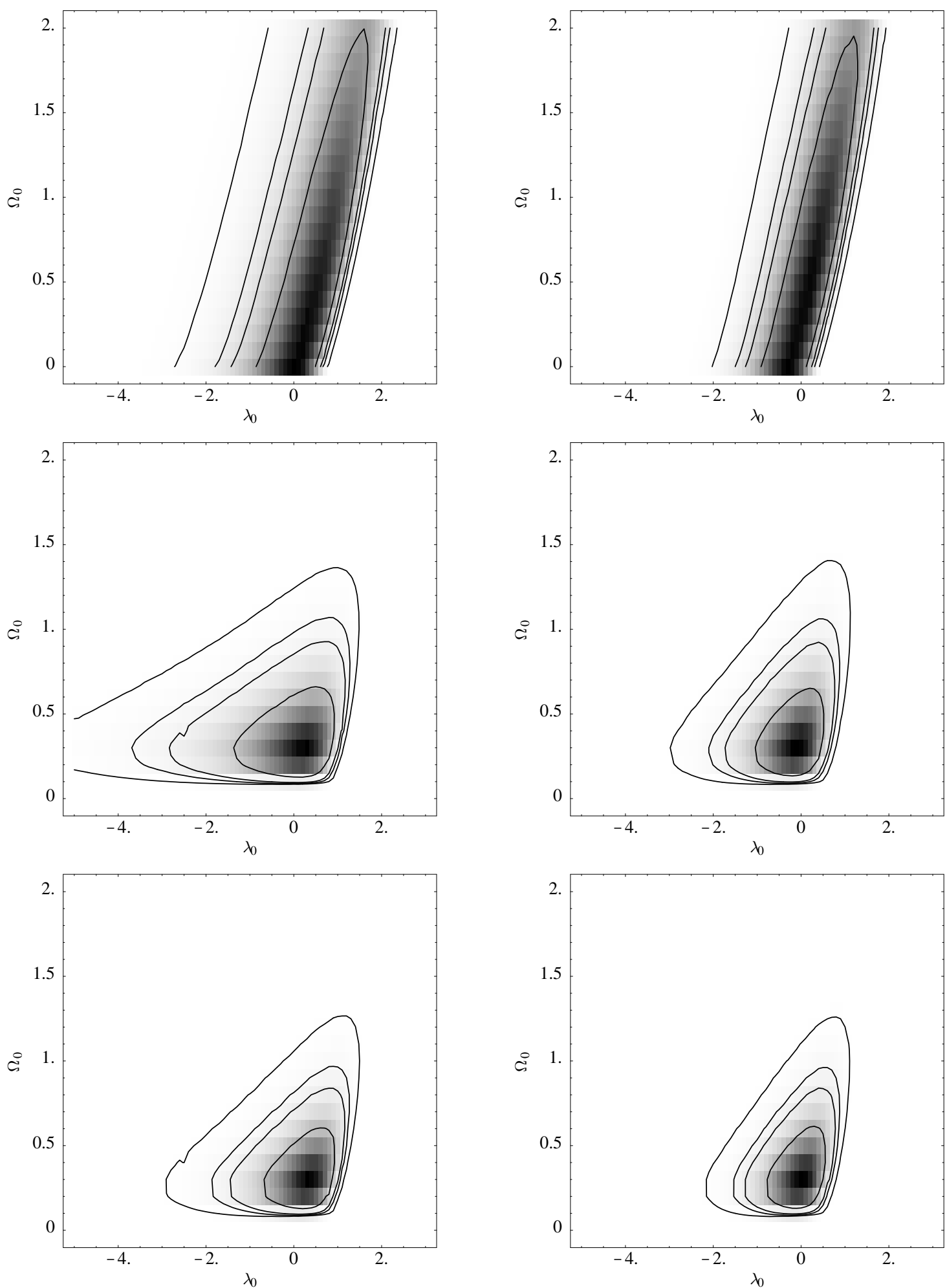

Fig. 3. Left column: The posterior probability density functions $p_{1}\left(\lambda_{0}, \Omega_{0} \mid D\right)$ (top panel), $p_{2}\left(\lambda_{0}, \Omega_{0} \mid D\right)$ (middle panel) and $p_{3}\left(\lambda_{0}, \Omega_{0} \mid D\right)$ (bottom panel). All nuisance parameters are assumed to take precisely their mean values. The pixel grey level is directly proportional to the likelihood ratio, darker pixels reflect higher ratios. The pixel size reflects the resolution of our numerical computations. The contours mark the boundaries of the minimum 0.68, 0.90, 0.95 and 0.99 confidence regions for the parameters $\lambda_{0}$ and $\Omega_{0}$. The respective amounts of information obtained from our sample data are $I_{1}=1.42, I_{2}=1.32$ and $I_{3}=1.45$. Right column: Exactly the same as the left panel, but the joint likelihood from the JVAS lens sample and the optical samples from Quast \& Helbig (1999). The respective amounts of information obtained from our joint sample data are $1.98,1.95$ and 1.96. See Paper I for definitions 

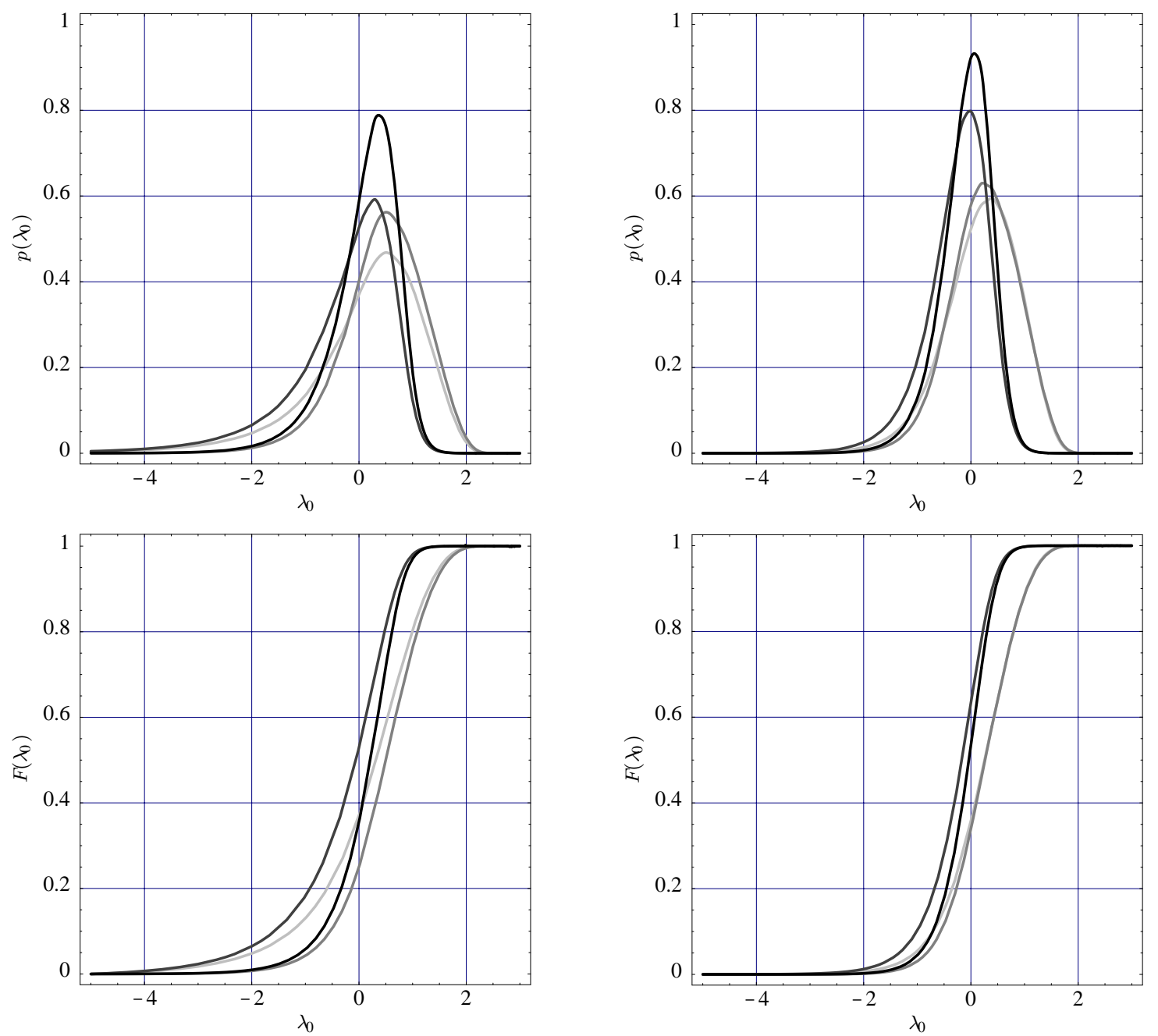

Fig. 4. Left column: The top panel shows the normalised marginal likelihood function $p\left(\lambda_{0} \mid D\right)$ (light gray curve) and the marginal posterior probability density functions $p_{1}\left(D \mid \lambda_{0}\right)$ (medium gray curve), $p_{2}\left(D \mid \lambda_{0}\right)$ (dark gray curve) and $p_{3}\left(D \mid \lambda_{0}\right)$ (black curve) derived from the JVAS analysis. All nuisance parameters are assumed to take precisely their mean values. The bottom panel shows the respective cumulative distribution functions. Right column: Exactly the same as the left panel, but the joint likelihood from the JVAS lens sample and the optical samples from Quast \& Helbig (1999)

Table 2. Marginal mean values, standard deviations and 0.95 confidence intervals for the parameter $\lambda_{0}$ on the basis of the marginal distributions shown in the top row of Fig. 4

\begin{tabular}{|c|c|c|c|c|c|c|}
\hline$\overline{\overline{\text { Sample }}}$ & 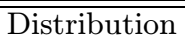 & Mean & standard deviation & \multicolumn{2}{|c|}{ 95\% c.l. range } & information \\
\hline$\overline{\text { JVAS }}$ & $p\left(D \mid \lambda_{0}\right)$ & 0.13 & 1.08 & -2.08 & 1.91 & \\
\hline JVAS & $p_{1}\left(\lambda_{0} \mid D\right)$ & 0.44 & 0.77 & -1.05 & 1.87 & 1.42 \\
\hline JVAS & $p_{2}\left(\lambda_{0} \mid D\right)$ & -0.29 & 0.98 & -2.38 & 1.17 & 1.32 \\
\hline JVAS & $p_{3}\left(\lambda_{0} \mid D\right)$ & 0.11 & 0.64 & -1.20 & 1.16 & 1.45 \\
\hline joint & $p\left(D \mid \lambda_{0}\right)$ & 0.19 & 0.70 & -1.17 & 1.48 & \\
\hline joint & $p_{1}\left(\lambda_{0} \mid D\right)$ & 0.24 & 0.63 & -0.96 & 1.46 & 1.98 \\
\hline joint & $p_{2}\left(\lambda_{0} \mid D\right)$ & -0.25 & 0.59 & -1.46 & 0.77 & 1.95 \\
\hline joint & $p_{3}\left(\lambda_{0} \mid D\right)$ & -0.09 & 0.48 & -1.08 & 0.77 & 1.96 \\
\hline
\end{tabular}


Table 3. Mean values and ranges for assorted confidence levels for the parameter $\lambda_{0}$ for our a priori and various a posteriori likelihoods from this work for $\Omega_{0}=0.3$. This should be compared to Table 3 in Paper I

\begin{tabular}{lccccccr}
\hline \hline Cosmological test & \multicolumn{2}{c}{$68 \%$ c.l. range } & \multicolumn{2}{c}{$90 \%$ c.l. range } & \multicolumn{2}{c}{$95 \%$ c.l. range } & \multicolumn{2}{c}{$99 \%$ c.l. range } \\
\hline JVAS, $p\left(D \mid \lambda_{0}\right)$ & -0.66 & 0.72 & -1.68 & 0.87 & -2.36 & 0.96 & -3.91 \\
JVAS, $p_{1}\left(\lambda_{0} \mid D\right)$ & -0.44 & 0.80 & -1.00 & 0.92 & -1.38 & 1.00 & -2.27 \\
JVAS, $p_{2}\left(\lambda_{0} \mid D\right)$ & -1.38 & 0.86 & -2.81 & 1.00 & -3.70 & 1.06 & $<-5.00$ \\
JVAS, $p_{3}\left(\lambda_{0} \mid D\right)$ & -0.69 & 0.86 & -1.45 & 0.99 & -1.89 & 1.03 & -2.91 \\
JVAS \& optical, $p\left(D \mid \lambda_{0}\right)$ & -0.54 & 0.26 & -1.08 & 0.44 & -1.41 & 0.54 & -2.15 \\
JVAS \& optical, $p_{1}\left(\lambda_{0} \mid D\right)$ & -0.63 & 0.40 & -0.95 & 0.53 & -1.18 & 0.62 & -1.72 \\
JVAS \& optical, $p_{2}\left(\lambda_{0} \mid D\right)$ & -1.02 & 0.44 & -1.72 & 0.63 & -2.08 & 0.72 & -2.95 \\
JVAS \& optical, $p_{3}\left(\lambda_{0} \mid D\right)$ & -0.77 & 0.52 & -1.23 & 0.63 & -1.52 & 0.70 & -2.15 \\
\hline
\end{tabular}

Table 4. Mean values and ranges for assorted confidence levels for the parameter $\lambda_{0}$ for our a priori and various a posteriori likelihoods from this work for $k=0$. This should be compared to Table 4 in Paper I

\begin{tabular}{|c|c|c|c|c|c|c|c|c|}
\hline Cosmological test & $68 \%$ & nge & $\overline{90 \%}$ & nge & $95 \% \mathrm{c.}$. & & $99 \%$ c. & \\
\hline$\overline{\text { JVAS, } p\left(D \mid \lambda_{0}\right)}$ & -0.11 & 0.70 & -0.83 & 0.78 & $<-1.00$ & 0.82 & $<-1.00$ & 0.86 \\
\hline JVAS, $p_{1}\left(\lambda_{0} \mid D\right)$ & 0.13 & 0.75 & -0.15 & 0.82 & -0.33 & 0.85 & -0.69 & 0.89 \\
\hline JVAS, $p_{2}\left(\lambda_{0} \mid D\right)$ & 0.35 & 0.77 & 0.13 & 0.83 & 0.02 & 0.85 & -0.21 & 0.88 \\
\hline JVAS, $p_{3}\left(\lambda_{0} \mid D\right)$ & 0.41 & 0.79 & 0.25 & 0.83 & 0.16 & 0.85 & -0.04 & 0.88 \\
\hline JVAS \& optical, $p\left(D \mid \lambda_{0}\right)$ & -0.15 & 0.45 & -0.49 & 0.55 & -0.69 & 0.60 & $<-1.00$ & 0.67 \\
\hline JVAS \& optical, $p_{1}\left(\lambda_{0} \mid D\right)$ & 0.02 & 0.54 & -0.12 & 0.61 & -0.29 & 0.64 & -0.60 & 0.70 \\
\hline JVAS \& optical, $p_{2}\left(\lambda_{0} \mid D\right)$ & 0.39 & 0.39 & 0.09 & 0.59 & 0.00 & 0.64 & $<-0.22$ & 0.70 \\
\hline JVAS \& optical, $p_{3}\left(\lambda_{0} \mid D\right)$ & 0.39 & 0.51 & 0.18 & 0.63 & 0.09 & 0.66 & -0.09 & 0.72 \\
\hline
\end{tabular}
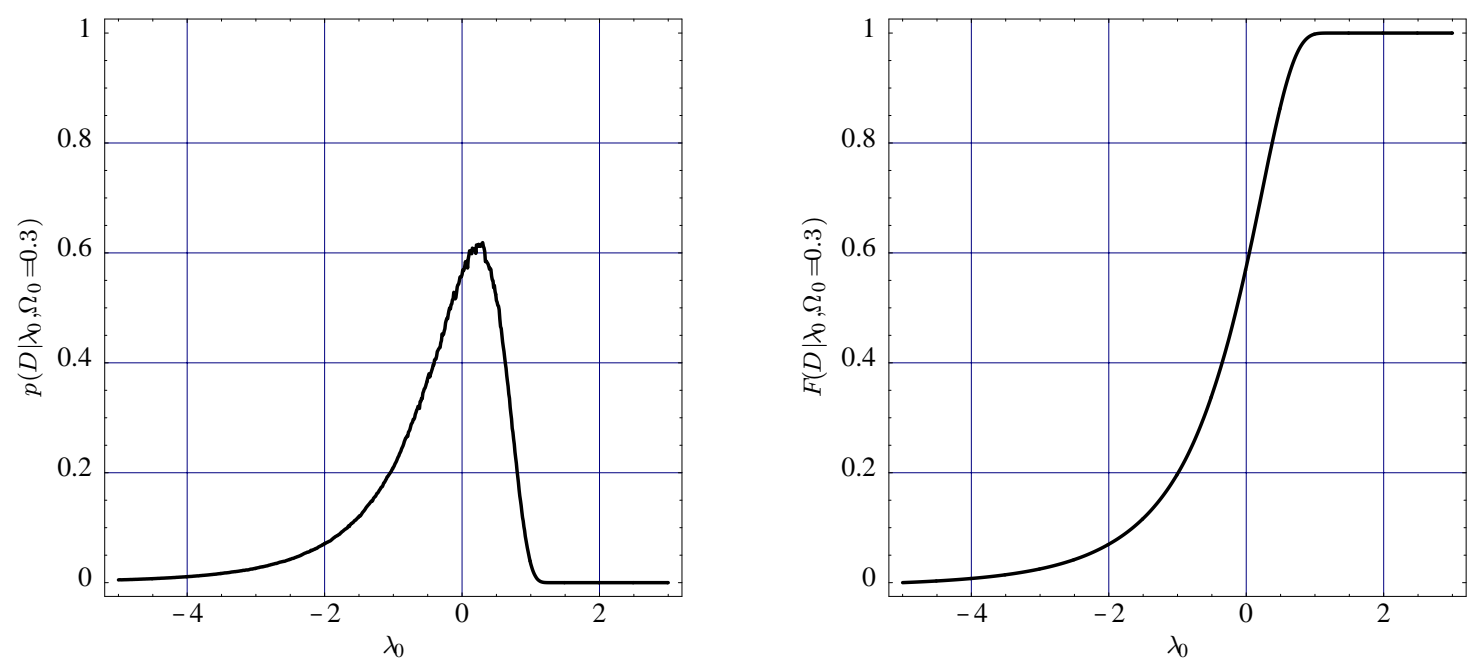

Fig. 5. Left panel: The likelihood function as a function of $\lambda_{0}$ for $\Omega_{0}=0.3$ and with all nuisance parameters taking their default values, using just the JVAS data. Right panel: The same but plotted cumulatively

Table 5. Confidence ranges for $\lambda_{0}$ assuming $\Omega_{0}=0.3$. Unlike the results presented in Table 3, these figures are for a specific value of $\Omega_{0}$ and not the values of intersection of particular contours with the $\Omega_{0}=0.3$ line in the $\lambda_{0}-\Omega_{0}$ plane. These are more appropriate if one is convinced that $\Omega_{0}=0.3$ and have been calculated using ten times better resolution than the rest of our results presented in this work. See Figs. 5 and 6

\begin{tabular}{lccccccc}
\hline \hline data set & \multicolumn{2}{c}{$68 \%$ c.l. range } & \multicolumn{2}{c}{$90 \%$ c.l. range } & \multicolumn{2}{c}{$95 \%$ c.l. range } & $99 \%$ c.l. range \\
\hline JVAS & -0.69 & 0.72 & -1.72 & 0.91 & -2.39 & 0.98 & -3.83 \\
JVAS+optical & -0.65 & 0.30 & -1.17 & 0.49 & -1.48 & 0.57 & -2.22 \\
\hline
\end{tabular}



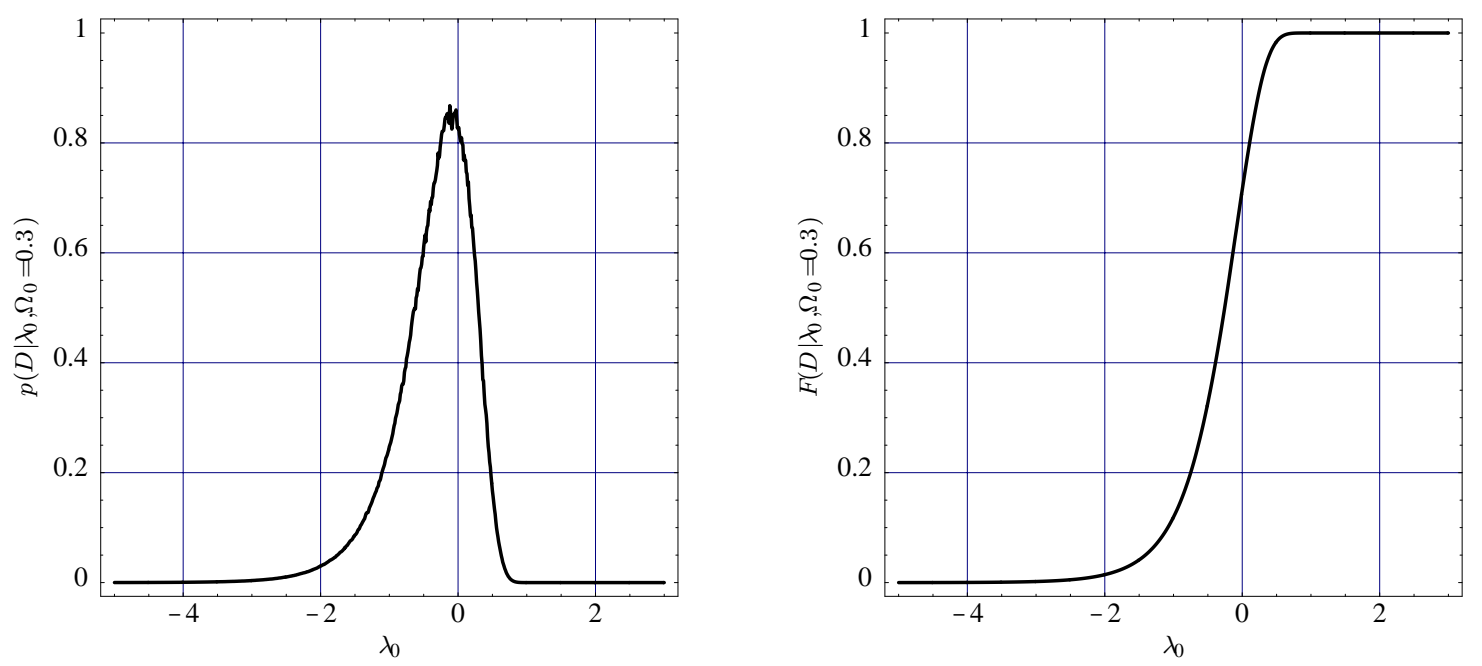

Fig. 6. As Fig. 5 but combining optical and radio data. Left panel: The likelihood function as a function of $\lambda_{0}$ for $\Omega_{0}=0.3$ and with all nuisance parameters taking their default values. Right panel: The same but plotted cumulatively

value of $\lambda_{0}$ from radio surveys, is what one would expect, as lens systems which go unnoticed will, all other things being equal, reduce the value of $\lambda_{0}$. This could be the case in optical surveys since it is possible that extinction in the lens galaxy and the fact that the resolution is only slightly better than the image separation could lead to lens systems being missed. Again, the general agreement does suggest though that these effects are not overwhelming.

Of course, one could imagine that the agreement is coincidental, the optical surveys being heavily affected by extinction and resolution bias and the radio surveys by our ignorance of the unlensed parent population. However, the fact that lens statistics in general gives results which are not in conflict with other cosmological tests (cf. Paper I) suggests that this is not the case. Moreover, extinction would bias the results from lens statistics and the $m-$ $z$ relation (e.g. for type Ia supernovae, cf. the results in Tables 3 and 4 of Paper I and in the references mentioned there) in the opposite direction. Thus, their agreement suggests that both methods have their systematics more or less under control.

The major source of uncertainty in radio lens surveys is the lack of knowledge about the redshift distribution and number-magnitude relation of the source sample (e.g. Kochanek 1996b). We are currently undertaking the necessary observations to reduce this source of systematic error. Since the time scale for this project is comparable to that for the followup of the CLASS survey, there seems little point in doing a better analysis of JVAS in the future, especially since CLASS is defined so that JVAS is essentially a subset of it ${ }^{1}$. The larger size of the CLASS survey, coupled with better knowledge of the redshift distribu-

1 The definition of both is flat-spectrum between $L$-band and $C$-band, i.e. $\alpha>-0.5$ where $s_{\mathrm{f}} \sim f^{\alpha}$, the essential difference being the lower flux density limit of $200 \mathrm{mJy}$ for JVAS and tion and number-magnitude relation of the source sample, should reduce both the random and systematic errors on our value of $\lambda_{0}$.

Acknowledgements. We thank our collaborators in the JVAS, CJF and CLASS surveys for useful discussions and for providing data in advance of publication and many colleagues at Jodrell Bank for helpful comments and suggestions. We also thank John Meaburn and Anthony Holloway at the Department of Astronomy in Manchester and the staff at Manchester Computing for providing us with additional computational resources. RQ is grateful to the CERES collaboration for making possible a visit to Jodrell Bank where this collaboration was initiated. This research was supported in part by the European Commission, TMR Programme, Research Network Contract ERBFMRXCT96-0034 "CERES".

\section{References}

Augusto P., Browne I.W.A., Wilkinson P.N., et al., 1999, MNRAS (in preparation)

Biggs A., Browne I.W.A., Helbig P., et al., 1998, MNRAS (in press) astro-ph/9811282

Browne I.W.A., Patnaik A.R., Wilkinson P.N., Wrobel J., 1998, MNRAS 293, 257

Condon J.J., Cotton W.D., Greisen E.W., et al., 1998, AJ 115, 1693

30 mJy for CLASS. However, since CLASS is defined based on newer catalogues (GB6 and NVSS: Gregory et al. 1996; Condon et al. 1998) than JVAS, there will be some essentially random differences due to differing quality of observations and variability of the sources. All the JVAS lenses mentioned in Table 1 are in the new CLASS sample, which, having no upper flux density limit, subsumes JVAS. The previous samples CLASS-I and CLASS-II will be similarly subsumed in the same sense as JVAS, though the differences here will be slightly larger since bands other than $L$ and $C$ were used in the preliminary definition of these samples. 
Cooray A.R., 1999, A\&A 342, 353

Cooray A.R., Quashnock J.M., Miller M.C., 1999, ApJ 511 562

Falco E., Kochanek C.S., Muñoz J.A., 1998, ApJ 494, 47 (FKM)

Fukugita M., Futamase T., Kasai M., 1990, MNRAS 246, 24

Fukugita M., Futamase K., Kasai M., Turner E.L., 1992, ApJ 393, 3

Gregory P.C., Scott W.K., Douglas K., Condon J.J., 1996, ApJS 103, 427

King L.J., Browne I.W.A., 1996, MNRAS 282, 67

King L.J., Jackson N.J., Blandford R.D., et al., 1998, MNRAS 295, L41

King L.J., Browne I.W.A., Marlow D.R., Patnaik A.R.,
Wilkinson P.N., 1999, MNRAS (in press)

Kochanek C.S., 1996a, ApJ 466, 638

Kochanek C.S., 1996b, ApJ 473, 595

Myers S.T., et al., 1999 (in preparation)

Patnaik A.R., Browne I.W.A., Wilkinson P.N., Wrobel J.M., 1992, MNRAS 254, 655

Quast R., Helbig P., 1999, A\&A 344 (Paper I)

Taylor G.B., Vermeulen R.C., Readhead A.C.S., et al., 1996, ApJS 107, 37

Turner E.L., 1990, ApJ 365, L43

Turner E.L., Ostriker J.P., Gott III J.R., 1984, ApJ 284, 1

Wilkinson P.N., Browne I.W.A., Patnaik A.R., Wrobel J., Sorothia B., 1998, MNRAS (in press) 